\title{
A randomized controlled cross-over trial and cost analysis comparing endoscopic ultrasound fine needle aspiration and fine needle biopsy
}

Authors

Institutions
A. Aziz Aadam ${ }^{1}$, Sachin Wani², Ashley Amick', Janak N. Shah ${ }^{3}$, Yasser M. Bhat ${ }^{3}$, Christopher M. Hamerski ${ }^{3}$, Jason B. Klapman ${ }^{4}$, V. Raman Muthusamy ${ }^{5}$, Rabindra R. Watson ${ }^{5}$, Alfred W. Rademaker ${ }^{6}$, Rajesh N. Keswani ${ }^{1}$, Laurie Keefer ${ }^{1}$, Ananya Das ${ }^{7}$, Srinadh Komanduri ${ }^{1}$

Institutions are listed at the end of article. accepted after revision 16. December 2015

\section{Bibliography}

Dol http://dx.doi.org/ 10.1055/s-0042-106958 Endoscopy International Open 2016; 04: E497-E505 (c) Georg Thieme Verlag KG Stuttgart · New York E-ISSN 2196-9736

Corresponding author Srinadh Komanduri, MD MS Division of Gastroenterology and Hepatology Feinberg School of Medicine Northwestern University 675 N. St. Clair Street Galter Pavilion 17-250 Chicago, IL 60611 koman1973@gmail.com submitted 16. July 2015

Background and study aims: Techniques to optimize endoscopic ultrasound-guided tissue acquisition (EUS-TA) in a variety of lesion types have not yet been established. The primary aim of this study was to compare the diagnostic yield (DY) of endoscopic ultrasound-guided fine needle aspiration (EUS-FNA) to endoscopic ultrasound-guided fine needle biopsy (EUS-FNB) for pancreatic and non-pancreatic masses.

Patients and methods: Consecutive patients referred for EUS-TA underwent randomization to EUS-FNA or EUS-FNB at four tertiary-care medical centers. A maximum of three passes were allowed for the initial method of EUS-TA and patients were crossed over to the other arm based on onsite specimen adequacy.

Results: A total of 140 patients were enrolled. The overall DY was significantly higher with speci-

\section{Introduction}

Endoscopic ultrasound-guided fine needle aspiration (EUS-FNA) is the standard of care for tissue sampling of pancreatic masses along with lesions within and adjacent to the gastrointestinal tract [1]. The diagnostic yield of EUS-FNA has been reported to be between $50 \%$ to $98 \%$ depending on the lesion type $[2,3]$. Techniques to optimize EUS-FNA in a variety of lesion types have not yet been established. Several technical variables have been studied, including needle gauge, use of stylet, and use of suction or capillary ("slow pull") techniques. While various observational studies have shown some impact on EUSFNA, alterations within these variables have not been demonstrated to consistently improve diagnostic yield [4-8]. In addition, EUS-FNA has limitations. The adequacy of a specimen may be dependent on the availability of on-site cytopathol-
* Meeting presentations: Digestive Disease Week, May 2014 mens obtained by EUS-FNB compared to EUSFNA $(90.0 \%$ vs. $67.1 \%, P=0.002)$. While there was no difference in the DY between the two groups for pancreatic masses (FNB: $91.7 \%$ vs. FNA: $78.4 \%, P=0.19)$, the DY of EUS-FNB was higher than the EUS-FNA for non-pancreatic lesions ( $88.2 \%$ vs. $54.5 \%, P=0.006$ ). Specimen adequacy was higher for EUS-FNB compared to EUS-FNA for all lesions $(P=0.006)$. There was a significant rescue effect of crossover from failed FNA to FNB in 27 out of 28 cases ( $96.5 \%, P=0.0003)$. Decision analysis showed that the strategy of EUS-FNB was cost saving compared to EUS-FNA over a wide range of cost and outcome probabilities.

Conclusions: Results of this RCT and decision analysis demonstrate superior DY and specimen adequacy for solid mass lesions sampled by EUSFNB.

ogy evaluation [9-13]. Tissue architecture and morphology is often difficult to maintain in EUSFNA samples [14-16]. This may limit immunohistochemistry or immunophenotyping, which is often helpful to establish a diagnosis for neoplasms such as lymphoma, metastasis, or subepithelial lesions [17-21].

Given these shortcomings, new approaches have shifted to the development of EUS-guided fine needle biopsy (EUS-FNB). Initial experience with a tru-cut (Quick Core ${ }^{\mathrm{TM}}$, Cook Medical, WinstonSalem, NC) biopsy needle was limited by the needle stiffness and difficulty with the firing mechanism [22]. More recently, a new generation of core biopsy needles have been introduced (Echotip Procore ${ }^{\mathrm{TM}}$, Cook Medical, Winston-Salem, NC). Multiple prospective cohort studies have suggested a significant improvement in diagnostic yield with EUS-FNB, while one RCT and some retrospective series have shown varying results [19, 22,23]. To date, there is limited published data comparing EUS-FNA and EUS-FNB with regard to diagnostic yield in pancreatic and non-pancreatic 
masses. In addition, the cost effectiveness of EUS-FNA and EUSFNB tissue acquisition techniques has not been previously evaluated. We hypothesized that EUS-FNB provides higher diagnostic yield than EUS-FNA. In this multicenter, prospective, randomized controlled trial, we aimed to compare the diagnostic yield between EUS-FNA and EUS-FNB in patients undergoing EUS-guided tissue acquisition (EUS-TA) for pancreatic and non-pancreatic masses.

\section{Patients and methods}

$\nabla$

\section{Study design}

This prospective, randomized, cross-over, multicenter trial was conducted at four tertiary-care medical centers: Northwestern Memorial Hospital, Chicago, IL; California Pacific Medical Center, San Francisco, CA; Moffitt Cancer Center, Tampa, FL; and University of California Los Angeles, Los Angeles, CA. The study was approved by the institutional review board at each participating center.

\section{Study population}

Consecutive patients referred for EUS-TA of solid masses (pancreas, lymph nodes, metastases, and subepithelial lesions) were prospectively enrolled from January 2013 through May 2014. The inclusion criteria included age greater than 18 years and presence of a solid mass lesion confirmed by at least one single investigational modality mainly computed tomography, magnetic resonance imaging, or endoscopy. Exclusion criteria were coagulopathy (INR $>1.5$ ), thrombocytopenia $(<50,000)$, episode of acute pancreatitis in the preceding 4 weeks, inability to safely perform EUS-TA, and refusal or inability to provide informed consent.

\section{EUS-FNA/FNB procedure}

All procedures were performed by experienced endosonographers, each of whom had performed more than 1000 cases. The curvilinear array echoendoscope (GF-UC140P or GF-UCT140, Olympus America, Center Valley, PA) was used in all cases.

EUS-FNA was performed using commercially available needles (Echotip $^{\mathrm{TM}}$, Cook Medical, Winston-Salem, NC; Expect ${ }^{\mathrm{TM}}$, Boston Scientific, Natick MA). Randomization of needle gauge, use of suction or "slow pull" technique, and use of stylet was not performed for this RCT and left to the discretion of the endosonographer. After the lesion was identified, it was punctured under Doppler guidance and approximately 10 to 15 back-and-forth movements were performed with the FNA needle into the target lesion using $10 \mathrm{~mL}$ of suction or the capillary "slow pull" technique.

EUS-FNB was performed using the (Echotip Procore ${ }^{\mathrm{TM}}$, Cook Medical, Winston-Salem, NC) needle. The Procore ${ }^{\mathrm{TM}}$ needle is composed of nitinol, contains a novel reverse cutting bevel design for procurement of a core specimen, and is available in 19-22-, and $25-\mathrm{G}$ sizes. The needle gauge was again left to the discretion of the endosonographer. For FNB, the capillary technique was used with a stylet (no syringe suction) with approximately 10 to 15 back-and-forth movements in the target lesion to acquire the core specimen. Endosonographers were not permitted to change needle gauge or use of suction/stylet during the 3 passes.

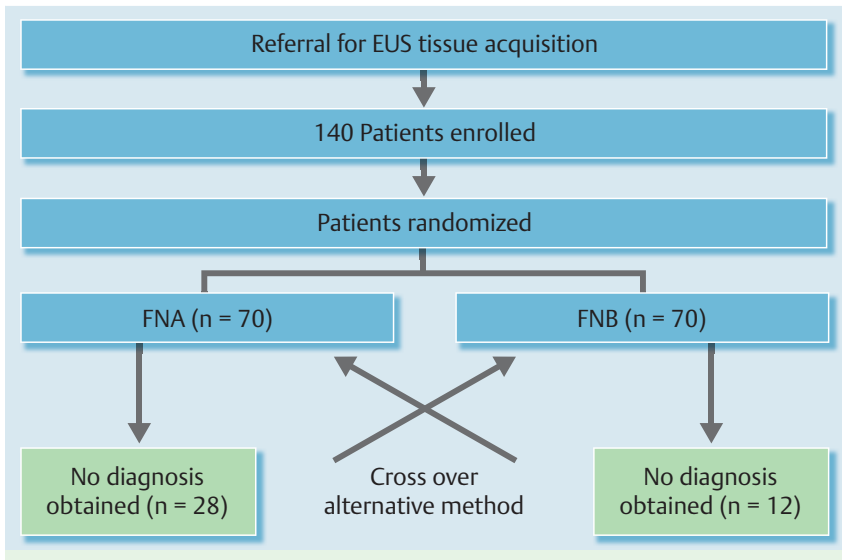

Fig. 1 Study flow chart

\section{Randomization}

Patients were centrally randomized to one of two arms: EUS-FNA or EUS-FNB. Randomization was performed by a computerized binary random number generator at the primary site (Northwestern University). The order of EUS-TA technique was determined using an opaque sealed envelope.

\section{Study protocol}

After randomization, a needle (FNA/FNB) was selected by the endosonographer. A maximum of three passes were allowed to obtain an adequate specimen as assessed by an on-site cytopathologist/cytotechnologist. If an adequate specimen was not obtained after three passes, the patient was crossed over to the alternate EUS-TA modality. An additional three passes were permitted in order to obtain an adequate specimen ( $\bullet$ Fig. $\mathbf{1})$.

\section{Cytopathologic and histologic assessment}

After tissue acquisition, the specimens were expressed onto a slide using a stylet and/or air flush. All FNA and FNB smears were prepared and assessed for adequacy on site by a cytotechnician/cytopathologist and subsequently confirmed by an experienced cytopathologist. Cytopathologists/cytotechnicans were not blinded to the tissue acquisition method due to the need for specialized preparation of the sample depending on the technique used. Furthermore, the acquisition of a tissue core by EUSFNB would be clearly visible, and lead to unblinding of the cytotechnician/cytopathologist. When performing EUS-FNA, one slide was air dried and prepared with Diff-Quik stain for on-site analysis. The second slide was fixed in alcohol solution to be stained later with Papanicolaou stain. Remaining FNA aspirate was placed into a standard cytologic solution for cell block preparation. When performing EUS-FNB, core specimens were prepared on slides using either smash or touch preparation according to the presence of fragmented tissue or a visible core specimen [24]. If the tissue acquired contained a visible core, a standard touch preparation was utilized. The touch preparation technique was performed by carefully placing the visible core specimen onto a slide and slowly moving the specimen around the slide before placing the specimen into a container with formalin for subsequent histologic evaluation. In the event that only fragmented or scant tissue was obtained, the smash protocol was performed: the tissue was put on a slide, and a second slide was used to gently crush the tissue between the two slides to prepare an air-dried crush preparation; any residual tissue was fixed in formalin for subsequent H\&E staining and histologic evaluation. 


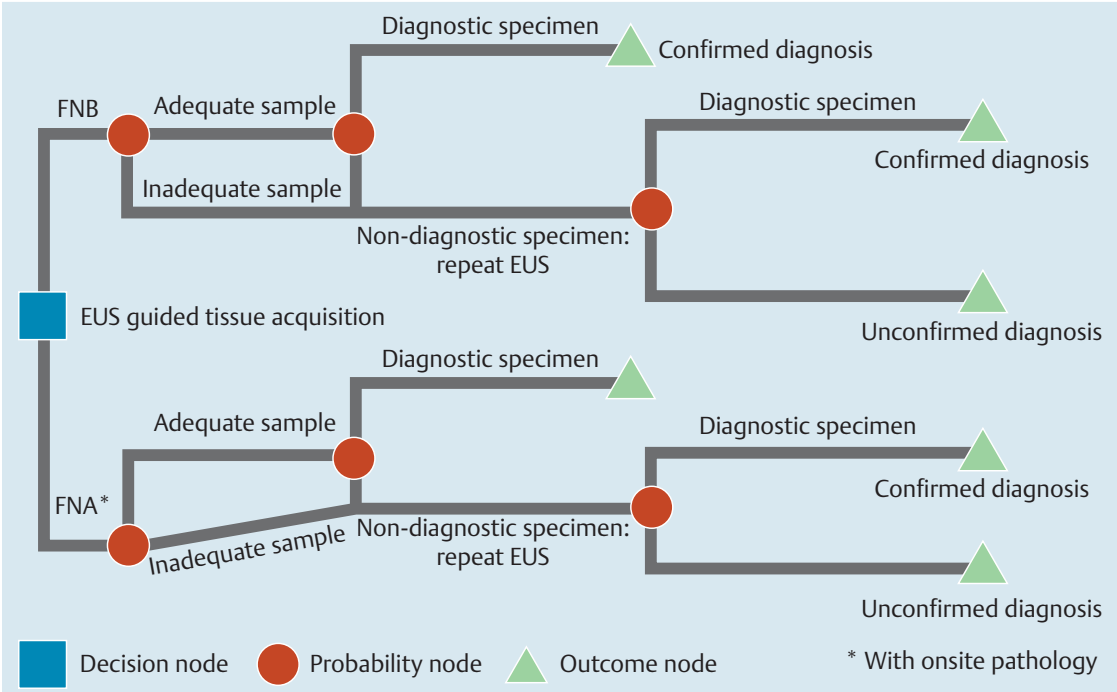

Fig. 2 Decision analysis tree showing the structure of the decision analysis model comparing the two competing strategies: EUS-FNB and EUS-FNA. In the decision tree, squares, circles, and triangles represent decision, probability and outcome nodes, respectively.

Each pass was assessed immediately for cellular adequacy and a final diagnosis was determined after review of all FNA or FNB material. Once an adequate specimen was obtained, the procedure was terminated. The final diagnosis was categorized as diagnostic or non-diagnostic (defined to include suspicious and atypical readings) and was standardized among all participating cytopathologists.

\section{Outcome measures and study definitions}

The primary aim of this study was to compare the diagnostic yield of EUS-FNA and EUS-FNB overall for all lesions. Our secondary aims included comparison of: (1) technical success; (2) diagnostic yield for pancreas and non-pancreas mass subgroups; (3) on-site specimen adequacy for EUS-FNA and EUS-FNB; (4) salvage effect of EUS-FNA and EUS-FNB; (5) cost-effectiveness analysis comparing EUS-FNA and EUS-FNB for pancreatic and nonpancreatic masses; and (6) adverse events. For this study we used the following standardized definitions: (1) diagnostic yield: percentage of the lesions sampled for which a tissue diagnosis is obtained; and (2) on-site specimen adequacy: the percentage of lesions sampled in which the obtained material is representative of the target site.

\section{Sample size and statistical analysis}

A sample size calculation was performed to conduct a betweensubjects comparison of EUS-FNA and EUS-FNB. Based on a literature review of EUS-FNA and EUS-FNB for heterogeneous indications, we expected a difference in diagnostic yield of $20 \%$ for all lesion types (pancreatic and non-pancreatic) between EUS-FNB (90\%) and EUS-FNA (70\%) after three needle passes. Using these criteria, a total of 140 patients were required with 70 patients in each arm. Results for continuous variables are expressed by using mean \pm standard deviation. Frequencies and percentages were calculated for categorical variables. Student's $t$-test was used to compare normally distributed continuous variables. Wilcoxon rank-sum test was used for variables not normally distributed and $\mathbf{X}^{2}$ analysis was used to compare the association between categorical variables and outcomes. McNemar test was used to compare paired binary data. A $P$ value $<0.05$ was considered significant and all statistical analyses were conducted using SAS version 9.2 (SAS Institute, Inc., Cary, North Carolina). The results are reported in accordance with the CONSORT statement [25].

\section{Decision analysis \\ $\nabla$}

A decision analysis tree was constructed using decision analysis software (TreeAge Pro, TreeAge Software, Williamstown, MA) and two competing strategies were evaluated from a third-party-payer perspective in a hypothetical cohort of patients with pancreatic and non-pancreatic masses undergoing EUS for EUSguided tissue acquisition ( $\bullet$ Fig. 2). Under strategy I, all patients underwent EUS-FNB without on-site cytopathology evaluation and specimens obtained were submitted to pathology for histologic evaluation. Under strategy II, all patients underwent EUSFNA and slides from each EUS FNA pass were prepared by an onsite cytology technician and a cytopathologist interpreted the slides immediately during the procedure to assess for adequacy and preliminary diagnosis. It was assumed that all cases were performed under monitored anesthesia care. In this decision analysis model, unlike the RCT, no cross-over design was followed. Under both strategies, repeat EUS was performed if clinically indicated in case the first EUS FNA/FNB was non-diagnostic. The model did not consider any cost associated with further workup beyond two non-diagnostic EUS procedures. Data on parameters such as diagnostic yield and adequacy of sampling with EUS-FNA and FNB and incremental diagnostic yield with second EUS procedures were obtained from results of this study and published data. The analysis was performed from a third-partypayer perspective, and Centers for Medicare and Medicaid Services (CMS) data on EUS FNA/FNB procedural reimbursement, procedural sedation costs with monitored anesthesia care, and pathology interpretation of on-site and off-site cytology slides were used. Cost parameters in terms of utilization and salary of the cytology technician were obtained from institutional data. Baseline estimates (data from the current randomized controlled trial and available literature) and costs were varied by using a sensitivity analysis through the ranges as shown in Table 1 [26-32]. Monte Carlo simulation analysis was performed in a hypothetical cohort of 1000 patients undergoing EUS and the incremental costs were calculated. 
Table 1 Relevant clinical probability estimates and costs used in the decision analysis.

\begin{tabular}{|c|c|c|}
\hline Clinical probabilities and costs & Baseline (range) & Source \\
\hline \multicolumn{3}{|l|}{ Cost variables } \\
\hline EUS FNA/FNB reimbursement & $\$ 1315(900-1500)$ & CMS \\
\hline Cost of FNB histology interpretation & $\$ 48(45-60)$ & CMS \\
\hline Cost of slide interpretation: onsite cytology & & CMS \\
\hline a) First slide & $\$ 45.58$ & \\
\hline b) Subsequent slide each & $\$ 20.56$ & \\
\hline Annual salary of cytology technician & $\$ 65,000(40,000-80,000)$ & Institutional data \\
\hline National CMS reimbursement 2013, Anesthesiologist per unit & $\$ 21.95$ & CMS \\
\hline \multicolumn{3}{|l|}{ Clinical probabilities } \\
\hline \multicolumn{3}{|l|}{ Number of passes } \\
\hline a) with EUS FNA and onsite cytology & $5(3-7)$ & {$[13,19,26]$} \\
\hline b) with EUS-FNB & 2 & [26] \\
\hline \multicolumn{3}{|l|}{ Pancreatic lesions } \\
\hline Probability of adequate sample with FNB & $0.81(0.54-0.9)$ & Current study, [23, 24, 27-29] \\
\hline Probability of adequate sample with FNA and onsite cytology evaluation & $0.65(0.5-0.95)$ & Current study, $[11,13]$ \\
\hline Diagnostic yield of malignancy with FNB & $0.92(0.7-0.95)$ & Current study, $[23,24,27-29]$ \\
\hline Diagnostic yield of malignancy with FNA & $0.78(0.55-0.85)$ & Current study, $[12,30]$ \\
\hline \multicolumn{3}{|l|}{ Non-pancreatic lesions } \\
\hline Probability of adequate sample with FNB & $0.82(0.54-0.9)$ & Current study, $[19,23,24,28,29]$ \\
\hline Probability of adequate sample with FNA and onsite cytology evaluation & $0.52(0.5-0.95)$ & Current study, $[12,31,32]$ \\
\hline Diagnostic yield of malignancy with FNB & $0.88(0.7-0.95)$ & Current study, $[19,23,24,28,29]$ \\
\hline Diagnostic yield of malignancy with FNA & $0.55(0.5-0.85)$ & Current study, $[12,31,32]$ \\
\hline
\end{tabular}

Table 2 Patient demographics and lesion characteristics.

\begin{tabular}{|llll|}
\hline Characteristic & $\begin{array}{l}\text { FNA } \\
(\mathbf{n = 7 0 )}\end{array}$ & $\begin{array}{l}\text { FNB } \\
(\mathbf{n = 7 0 )}\end{array}$ & P value \\
\hline Mean age (SD) & $63.7(14.4)$ & $64.0(14.4)$ & 0.88 \\
\hline Male (n, \%) & $34(48.6)$ & $40(57.1)$ & 0.24 \\
\hline Caucasian (n, \%) & $43(61.4)$ & $44(62.9)$ & 0.13 \\
\hline Mean lesion size mm (SD) & $30.2(18.7)$ & $29.2(14.1)$ & 0.71 \\
\hline Pancreatic masses (n, \%) & $37(52.9)$ & $36(51.4)$ & 0.99 \\
\hline Non-pancreatic masses (n, \%) & $33(47.1)$ & $34(48.6)$ & 0.98 \\
\hline Thoracic/abdominal/pelvic mass & 16 & 15 & 0.78 \\
\hline Lymphadenopathy & 10 & 11 & 0.88 \\
\hline Subepithelial lesions & 7 & 8 & 0.67 \\
\hline
\end{tabular}

\section{Results}

$\nabla$

\section{Patients}

A total of 140 (70 EUS-FNA, 70 EUS-FNB) consecutive subjects were enrolled in the study over a 17-month period. Seventyfour patients $(52.8 \%$ ) were male. There were no differences in patient demographics and lesion characteristics between the two groups. Furthermore, pancreatic and non-pancreatic lesions were distributed evenly between EUS-FNA and EUS-FNB ( $\bullet$ Table 2).

\section{Tissue acquisition techniques}

EUS-TA was technically successful in all patients without any adverse events. For patients randomized to the EUS-FNA arm, a 22 G needle was used in 48 (68.6\%) while a $25 \mathrm{G}$ needle was used in 22 (31.4\%). For patients randomized to the EUS-FNB arm, a $19 \mathrm{G}$ needle was used in $7(10 \%)$, a $22 \mathrm{G}$ needle in $37(52.8 \%)$, and a $25 \mathrm{G}$ needle in 26 (37.1\%). There was no significant difference in needle gauge or the number of passes performed (FNA mean \pm [SD] $3.0 \pm(1.0)$, FNB mean $\pm[$ [SD] $2.8 \pm(1.0), P=0.20)$ with FNA and FNB techniques ( $\triangle$ Table 3 ).

\section{Diagnostic yield}

The overall DY was significantly higher with specimens obtained by EUS-FNB compared to EUS-FNA ( $90 \%$ vs. $67.1 \%, P=0.002$ ). Non-pancreatic lesions were associated with a higher diagnostic yield with the FNB technique compared to FNA ( $88.2 \%$ vs. $54.5 \%$, $P=0.006$ ). There was no statistically significant difference between EUS-FNA and EUS-FNB for pancreas masses (91.7\% vs. $78.4 \%, P=0.19$ ). The differences observed were independent of lesion size, number of passes, use of suction or stylet, or needle gauge ( $\bullet$ Table 3 ). Final diagnosis by lesion type is listed in supplementary $\bullet$ Table 1.

\section{Specimen adequacy}

The overall specimen adequacy was significantly greater for EUSFNB compared to EUS-FNA ( $82.8 \%$ vs. $60.0 \%, P=0.006$ ). Greater specimen adequacy was observed in non-pancreatic lesions sampled by EUS-FNB ( $82.4 \%$ vs. $51.5 \%, P=0.019)$. There was no statistically significant difference in specimen adequacy for pancreatic masses between EUS-FNA and EUS-FNB $(83.3 \%$ vs. $67.5 \%, P=$ 0.19).

\section{Crossover salvage effect}

We also evaluated the salvage effect of the alternative tissue acquisition method when the initial three passes with either EUSFNA or EUS-FNB failed to provide an adequate specimen. There was a significant salvage effect in diagnostic yield for crossover from failed EUS-FNA to EUS-FNB in 27 out of 28 cases ( $96.4 \%, P=$ 0.0003 ). This effect was independent of lesion subtype (15 nonpancreatic vs. 12 pancreatic, $P=0.12$ ). In contrast, a diagnosis was established in only $5 / 12$ cases $(41.7 \%, P=0.99)$ of failed EUS-FNB that were crossed over to EUS-FNA. This effect was also independent of lesion subtype ( 3 non-pancreatic vs. 2 pancreatic, $P=$ $0.76)$. 


\begin{tabular}{|c|c|c|c|}
\hline Characteristic & $\begin{array}{l}\text { FNA } \\
(n=70)\end{array}$ & $\begin{array}{l}\text { FNB } \\
(n=70)\end{array}$ & $P$ value \\
\hline Mean no. of passes mean (SD) & $3.0(1.0)$ & $2.8(1.0)$ & 0.20 \\
\hline Needle Size (n, \%) & & & 0.051 \\
\hline 19-G & $0(0)$ & $7(10)$ & \\
\hline $22-G$ & $48(68.6)$ & $37(52.9)$ & \\
\hline $25-G$ & $22(31.4)$ & $26(37.1)$ & \\
\hline Diagnostic yield (n, \%) & $47 / 70(67.1)$ & $63 / 70(90)$ & 0.002 \\
\hline Pancreatic & $29 / 37(78.4)$ & $33 / 36(91.7)$ & 0.19 \\
\hline Non-pancreatic & $18 / 33(54.5)$ & $30 / 34(88.2)$ & 0.006 \\
\hline Specimen adequacy (n, \%) & $42 / 70(60.0)$ & $58 / 70(82.8)$ & 0.006 \\
\hline Pancreatic & $25 / 37(67.5)$ & $30 / 36(83.3)$ & 0.19 \\
\hline Non-pancreatic & $17 / 33(51.5)$ & $28 / 34(82.4)$ & 0.019 \\
\hline \multicolumn{4}{|l|}{ Crossover diagnostic yield (n, \%) } \\
\hline FNA to FNB $(n=28)$ & & $27(96.4 \%)$ & 0.0003 \\
\hline FNB to FNA $(n=12)$ & $5(41.7) \%$ & & 0.99 \\
\hline
\end{tabular}

Table 3 Summary of tissue acquisition results.

\begin{tabular}{|c|c|c|}
\hline Baseline analysis & Cost (\$) per procedure & Incremental cost \\
\hline \multicolumn{3}{|l|}{ Pancreatic lesions } \\
\hline FNB & $\$ 1926$ & \\
\hline FNA with on-site cytopathology & $\$ 2538$ & $\$ 612$ \\
\hline \multicolumn{3}{|l|}{ Non-pancreatic lesions } \\
\hline FNB & $\$ 1931$ & \\
\hline FNA with on-site cytopathology & $\$ 2926$ & $\$ 995$ \\
\hline
\end{tabular}

Table 4 Results of baseline analysis.

\section{Decision analysis results}

The results of baseline analysis are shown in 1 Table4. Comparing the two strategies for pancreatic and non-pancreatic masses, strategy II of EUS-FNA was dominated by strategy I of EUS-FNB in that it was more expensive. The results of the Monte Carlo analysis for pancreatic masses showed that under strategy I, EUS-FNB confirmed diagnosis in 988 patients at an average cost of $\$ 2,152$ (95\% CI, 2070-2162) per patient and under strategy II, EUS-FNA confirmed diagnosis in 921 patients at an average cost of $\$ 2,605$ (95\% CI, 2263 -2664) per patient. The results of the Monte Carlo analysis for non-pancreatic masses showed that under strategy I, EUS-FNB confirmed diagnosis in 991 patients at an average cost of $\$ 1921$ (95\% CI, 1874-1968) per patient and under strategy II, EUS-FNA confirmed diagnosis in 725 patients at an average cost of $\$ 2942$ (95\% CI, $2901-2985)$ per patient. Supplementary Fig. 1 is a Tornado diagram showing the results of one-way sensitivity analyses of the important variables impacting the outcomes of the decision analysis. Besides the costs of the EUS procedure and sedation, sample adequacy and diagnostic yield of EUS-FNB had the most influence on the results. $\bullet$ Supplementary Fig. $\mathbf{2} \mathbf{a}$ and $\mathbf{b}$ show the results of the two-way sensitivity analyses when probability of sample adequacy and diagnostic yield with both tissue sampling techniques are varied simultaneously. The robustness of the results of this decision analysis is highlighted by the analyses (with all the reported values of adequacy of sampling and diagnostic yield from the current study and published data) and suggests that EUS-FNB is potentially more economical compared to EUS-FNA.

\section{Discussion}

The optimal EUS-TA technique has not been clearly defined. This gap is further underscored by the lower DY for non-pancreatic mass lesions. However, recent data suggest that EUS-FNB may improve DY [23,33-35]. In this multicenter, randomized controlled trial with crossover design, we compared the diagnostic yield of EUS-FNA and EUS-FNB in pancreatic and non-pancreatic mass lesions. Results of this study demonstrated a significantly higher overall DY of EUS-FNB over EUS-FNA in non-pancreatic lesions.

Data are limited from randomized controlled trials comparing EUS-FNA to EUS-FNB in non-pancreatic mass lesions. [35,36] The published diagnostic yield of EUS-FNA for gastric subepithelial masses ranges from $42 \%$ to $92 \%[37,38]$. Recently, Kim et al. conducted a randomized controlled trial of 22 patients with gastric subepithelial tumors. Patients who were randomized to EUSFNB had significantly higher diagnostic yield compared to patients who underwent EUS-FNA (75\% vs. $20 \%, P=0.010$ ) [28]. Previously published prospective studies have also noted high diagnostic accuracy of the FNB technique in non-pancreatic mass lesions $[23,35]$. A recent study evaluated 125 patients with nonpancreatic masses using the $22-\mathrm{G}$ core biopsy needle. They demonstrated a diagnostic yield of $83 \%$ [34]. In another prospective cohort study, Iglesias-Garcia and colleagues evaluated the performance of the 19-G FNB needle in 114 patients. They reported a diagnostic accuracy of $83.5 \%$ in the 67 patients with non-pancreatic lesions [23]. Our study results indicate a significantly greater diagnostic yield with EUS-FNB of non-pancreatic lesions of $88.2 \%$ compared to $54.5 \%$ with EUS-FNA $(P=0.006)$, suggesting that EUS-FNB is the optimal modality for tissue acquisition in non-pancreatic masses.

The role of EUS-FNA for pancreatic mass lesions is well established with diagnostic yield greater than 90\% [39]. The pooled sensitivity from five meta-analyses on EUS-FNA for solid pancreatic mass lesions is $85 \%$ to $89 \%$, with higher diagnostic accuracy in prospective, multicenter studies [10,40-42]. In addition, prior prospective studies comparing FNA and FNB techniques for pancreatic mass lesions have failed to show a benefit for the FNB technique. In another prospective, randomized, controlled trial of 28 patients, Bang and colleagues evaluated the efficacy of a 22-G FNA and FNB needle [19]. They found no significant differ- 
ence in diagnostic yield or number of passes required to obtain a diagnosis between the two techniques. However, this study was limited by a very small sample size. In a prospective cohort study of 32 patients with solid pancreatic masses comparing FNA cytology to FNB core histology, Strand et al. actually found that the FNB technique with a $22-\mathrm{G}$ needle was associated with a significantly reduced diagnostic yield compared to FNA (FNA: 93.8\%, FNB: $28.1 \%, P<0.001$ ) [43]. However, this study used suction during procurement of FNB, which may have increased the bloodiness and contamination of specimens. Two recently published prospective, randomized trials have also shown no difference in diagnostic yield between EUS-FNA and EUS-FNB for pancreas mass lesions $[44,45]$. Consistent with the previously published literature, results from this randomized controlled trial also showed no difference in diagnostic yield between EUS-FNA and EUS-FNB for pancreatic masses (FNB: $91.7 \%$, FNA: $78.4 \%, P=$ $0.19)$.

False-negative diagnoses during EUS-FNA have been reported in $4 \%$ to $45 \%$ of solid pancreatic masses and $6 \%$ to $14 \%$ of lymph nodes [46]. False-negative cytology is most often due to inaccurate tissue sampling, lesion characteristics (e.g. necrosis), insufficient endosonographer experience, or misinterpretation of specimens [3]. Our study not only highlights the value of EUS-FNB in non-pancreatic lesions, but also demonstrates the value of an FNB specimen as salvage for inadequate FNA. Furthermore, this effect was not dependent on lesion subtype. Therefore, it is important to recognize the lesion characteristics that may pose a challenge to obtaining a tissue diagnosis and use the most appropriate tissue acquisition technique.

Using probabilities from published data and results from this randomized controlled trial, a cost-effectiveness analysis from a societal perspective showed that EUS-FNB (strategy of EUSFNB - two passes without on-site cytopathology evaluation) was more cost-effective than EUS-FNA (strategy of FNA - passes dictated by on-site cytopathology evaluation) of pancreatic and non-pancreatic masses. While the strategy of EUS-FNB was more cost effective, the authors acknowledge that the decision analysis does not mirror the methodology of the current randomized controlled trial. The strategies used in this decision model reflect current clinical practice with regard to the use of these EUS tissue acquisition techniques. These results were even more pronounced when probabilities regarding specimen adequacy and diagnostic yield from this study were used for the decision model. Results from the Monte Carlo analysis and sensitivity analysis confirmed the above results. Variables with the maximal impact on the results were cost of EUS procedure and sedation, specimen adequacy, and diagnostic yield associated with EUSFNB.

Our study has several inherent strengths. The study was designed as a prospective, multicenter, randomized controlled trial, thereby minimizing selection and assignment bias. Our sample size was heterogeneous with nearly proportionate number of pancreatic and non-pancreatic masses. There are a few limitations to this study that warrant mention. Patients in the study were not followed longitudinally and, in the absence of a gold standard reference of surgical specimens, accuracy rates could not be determined. Previous data have established the optimal number of passes as seven for EUS-FNA without on-site cytopathology, and three in the presence of on-site cytopathology $[6,47]$. No further significant yield was demonstrated above these threshold numbers. As a result, we allotted three passes per technique prior to crossover. This is potentially responsible for the lower specimen adequacy and DY of EUS-FNA for pancreatic and non-pancreatic masses than what is seen in clinical practice. However, given the standardization across both arms, the effect of this design on the difference in DY is likely minimal [13]. Our study was powered to compare diagnostic yield for EUS-FNA and EUS-FNB for pancreatic and non-pancreatic masses combined. Therefore, it was underpowered to detect differences for the subgroup of pancreatic mass lesions, leading to a possible type II error. Our diagnostic yield of EUS-FNA of $78 \%$ for pancreatic lesions is lower than reported in recent randomized trials [19]. However, studies with comparable methodologies have reported similar diagnostic yield [48]. Furthermore, our DY for EUS-FNA is higher than the recommended threshold of $70 \%$ for quality indicators in EUS [49]. The FNA technique and use of needle gauge was not standardized in our study, but rather, left at the discretion of the endosonographer in this multicenter RCT in order to best simulate true clinical practice in addition to the fact that there is lack of level 1 evidence that these variables significantly affect the diagnostic yield. Meta-analysis data has demonstrated increased diagnostic yield of 25-G needles for pancreatic mass lesions, howeve, $r$ in our RCT there was no significant difference between needle gauge in the FNA and FNB groups in our study, thus eliminating any potential bias or benefit in either group $[30,50]$. Our study was performed at high-volume tertiary centers, therefore, there was a bias toward malignant lesions. EUS-FNA was performed in patients with a high pretest probability of malignancy (mass on cross sectional imaging, jaundice, weight loss, elevated CA 19-9) as there is increasing utilization of neoadjuvant chemotherapy in the United States which requires a tissue diagnosis. This may limit the broad application of our results to practice settings outside of tertiary centers in the United States.

Lastly, it was not possible to mirror the methodology of the decision analysis with that of the randomized controlled trial, however, the decision analysis adds credence to the RCT results and our recommendations.

In summary, the current RCT demonstrates superior diagnostic yield of EUS-FNB over EUS-FNA. The difference was primarily due to the significantly greater DY of EUS-FNB for non-pancreatic masses. Our results also provide further evidence for the continued use of EUS-FNA for tissue acquisition in pancreatic mass lesions. However, EUS-FNB should be considered as the initial sampling technique for non-pancreatic masses and as a salvage technique when on-site assessment of cytology samples is inadequate. Based on these results, the investigators propose an algorithmic approach to EUS-TA in patients with pancreatic and nonpancreatic masses [3]. These results and the approach to EUS-TA need to be validated in future prospective, multicenter, randomized controlled trials.

Competing interests: Drs. Muthusamy and Komanduri are consultants for Boston Scientific and Covidien; Dr. Bhat is a consultant for Covidien; Dr. Das has received research support from RedPath Integrated Pathology and is a consultant for Boston Scientific; Dr. Wani is a consultant for Covidien and has received Educational Grant support from Cook Medical. 
Institutions

${ }^{1}$ Division of Gastroenterology and Hepatology, Northwestern University, Chicago, Illinois, United States

2 Division of Gastroenterology and Hepatology, University of Colorado Anschutz Medical Center, Aurora, Colorado, United States

${ }^{3}$ Paul May and Frank Stein Interventional Endoscopy Center, California Pacific Medical Center, San Francisco, California, United States

${ }^{4}$ Division of Gastroenterology, Moffitt Cancer Center, Tampa, Florida, United States

${ }^{5}$ Division of Digestive Diseases, Department of Medicine, David Geffen School of Medicine at University of California, Los Angeles (UCLA), Los Angeles, California, United States

${ }^{6}$ Department of Preventative Medicine, Northwestern University, Chicago, Illinois, United States

${ }^{7}$ Arizona Digestive Health, Gilbert, Arizona, United States

\section{References}

1 Early DS, Ben-Menachem T. ASGE Standards of Practice Committee. et al. Appropriate use of GI endoscopy. Gastrointest Endosc 2012; 75: $1127-1131$

2 Dumonceau J-M, Polkowski M, Larghi A et al. Indications, results, and clinical impact of endoscopic ultrasound (EUS)-guided sampling in gastroenterology: European Society of Gastrointestinal Endoscopy (ESGE) Clinical Guideline. Endoscopy 2011; 43: 897-912

3 Wani S, Muthusamy VR, Komanduri S. EUS-guided tissue acquisition: an evidence-based approach (with videos). Gastrointest Endosc 2014; 80: $939-959$

4 Siddiqui UD, Rossi F, Rosenthal LS et al. EUS-guided FNA of solid pancreatic masses: a prospective, randomized trial comparing 22-gauge and 25-gauge needles. Gastrointest Endosc 2009; 70: 1093 -1097

5 Camellini L, Carlinfante G, Azzolini $F$ et al. A randomized clinical trial comparing $22 \mathrm{G}$ and $25 \mathrm{G}$ needles in endoscopic ultrasound-guided fine-needle aspiration of solid lesions. Endoscopy 2011; 43: 709-715

6 Wani S, Early D, Kunkel J et al. Diagnostic yield of malignancy during EUS-guided FNA of solid lesions with and without a stylet: a prospective, single blind, randomized, controlled trial. Gastrointest Endosc 2012; 76: 328-335

7 Lee JK, Choi JH, Lee KH et al. A prospective, comparative trial to optimize sampling techniques in EUS-guided FNA of solid pancreatic masses. Gastrointest Endosc 2013; 77: 745-751

8 Bang JY, Ramesh J, Trevino J et al. Objective assessment of an algorithmic approach to EUS-guided FNA and interventions. Gastrointest Endosc 2013; 77: 739-744

9 Alsohaibani F, Girgis S, Sandha GS. Does onsite cytotechnology evaluation improve the accuracy of endoscopic ultrasound-guided fine-needle aspiration biopsy? Can J Gastroenterol 2009; 23: 26-30

10 Hébert-Magee S, Bae S, Varadarajulu $S$ et al. The presence of a cytopathologist increases the diagnostic accuracy of endoscopic ultrasoundguided fine needle aspiration cytology for pancreatic adenocarcinoma: a meta-analysis. Cytopathology 2013; 24: 159-171

11 Iglesias-Garcia J, Dominguez-Munoz JE, Abdulkader I et al. Influence of on-site cytopathology evaluation on the diagnostic accuracy of endoscopic ultrasound-guided fine needle aspiration (EUS-FNA) of solid pancreatic masses. Am J Gastroenterol 2011; 106: 1705-1710

12 Klapman JB, Logrono R, Dye CE et al. Clinical impact of on-site cytopathology interpretation on endoscopic ultrasound-guided fine needle aspiration. Am J Gastroenterol 2003; 98: 1289-1294

13 Wani S, Mullady D, Early DS et al. Clinical impact of immediate on-site cytopathology (CyP) evaluation during endoscopic ultrasound-guided fine needle aspiration (EUS-FNA) of pancreatic mass: Interim analysis of a multicenter randomized con- trolled trial. Gastrointest Endosc 2013; 77: 2

14 Wiersema MJ, Vilmann P, Giovannini M et al. Endosonography-guided fine-needle aspiration biopsy: diagnostic accuracy and complication assessment. Gastroenterology 1997; 112: 1087-1095

15 Ribeiro A, Vazquez-Sequeiros E, Wiersema LM et al. EUS-guided fineneedle aspiration combined with flow cytometry and immunocytochemistry in the diagnosis of lymphoma. Gastrointest Endosc 2001; 53: $485-491$

16 Erickson RA, Sayage-Rabie L, Beissner RS. Factors predicting the number of EUS-guided fine-needle passes for diagnosis of pancreatic malignancies. Gastrointest Endosc 2000; 51: 184-190

17 Levy MJ, Wiersema MJ. EUS-guided Trucut biopsy. Gastrointest Endosc 2005; $62: 417-426$
18 Jenssen $C$, Dietrich $C F$. Endoscopic ultrasound-guided fine-needle aspiration biopsy and trucut biopsy in gastroenterology - An overview. Best Pract Res Clin Gastroenterol 2009; 23: 743 - 759

19 Bang JY, Hebert-Magee S, Trevino J et al. Randomized trial comparing the 22-gauge aspiration and 22-gauge biopsy needles for EUS-guided sampling of solid pancreatic mass lesions. Gastrointest Endosc 2012; 76: $321-327$

20 Bhutani MS, Gress FG, Giovannini M et al. The No Endosonographic Detection of Tumor (NEST) Study: a case series of pancreatic cancers missed on endoscopic ultrasonography. Endoscopy 2004; 36: 385 - 389

21 Varadarajulu S, Tamhane A, Eloubeidi MA. Yield of EUS-guided FNA of pancreatic masses in the presence or the absence of chronic pancreatitis. Gastrointest Endosc 2005; 62: 728 - 736

22 Gerke H, Rizk MK, Vanderheyden $A D$ et al. Randomized study comparing endoscopic ultrasound-guided Trucut biopsy and fine needle aspiration with high suction. Cytopathology 2010; 21: $44-51$

23 Iglesias-Garcia J, Poley J-W, Larghi A et al. Feasibility and yield of a new EUS histology needle: results from a multicenter, pooled, cohort study. Gastrointest Endosc 2011; 73: 1189-1196

24 Krishnan K, Dalal S, Nayar $R$ et al. Rapid on-site evaluation of endoscopic ultrasound core biopsy specimens has excellent specificity and positive predictive value for gastrointestinal lesions. Dig Dis Sci 2013; 58: $2007-2012$

25 Schulz KF, Altman DG, Moher D et al. CONSORT 2010 statement: updated guidelines for reporting parallel group randomized trials. Ann Intern Med 2010; 152: 726-732

26 Iwashita T, Nakai Y, Samarasena JB et al. High single-pass diagnostic yield of a new 25-gauge core biopsy needle for EUS-guided FNA biopsy in solid pancreatic lesions. Gastrointest Endosc 2013; 77: 909-915

27 Larghi A, Iglesias-Garcia J, Poley J-W et al. Feasibility and yield of a novel 22-gauge histology EUS needle in patients with pancreatic masses: a multicenter prospective cohort study. Surg Endosc 2013; 27: $3733-$ 3738

28 Hucl T, Wee E, Anuradha $S$ et al. Feasibility and efficiency of a new $22 \mathrm{G}$ core needle: a prospective comparison study. Endoscopy 2013; 45: $792-798$

29 Keswani RN, Krishnan K, Wani S et al. Addition of Endoscopic Ultrasound (EUS)-guided fine needle aspiration and on-site cytology to EUS-guided fine needle biopsy increases procedure time but not diagnostic accuracy. Clin Endosc 2014; 47: $242-247$

30 Madhoun MF, Wani SB, Rastogi A et al. The diagnostic accuracy of 22gauge and 25-gauge needles in endoscopic ultrasound-guided fine needle aspiration of solid pancreatic lesions: a meta-analysis. Endoscopy 2013; 45: 86-92

31 Cleveland P, Gill KRS, Coe SG et al. An evaluation of risk factors for inadequate cytology in EUS-guided FNA of pancreatic tumors and lymph nodes. Gastrointest Endosc 2010; 71: 1194-1199

32 Ecka RS, Sharma M. Rapid on-site evaluation of EUS-FNA by cytopathologist: an experience of a tertiary hospital. Diagn Cytopathol 2013; 41: $1075-1080$

33 Witt BL, Adler DG, Hilden K et al. A comparative needle study: EUS-FNA procedures using the HD ProCore ${ }^{\mathrm{TM}}$ and EchoTip $^{\circledR}$ 22-gauge needle types. Diagn Cytopathol 2013; 41: 1069-1074

34 Paik WH, Park Y, Park DH et al. Prospective evaluation of new 22 gauge endoscopic ultrasound core needle using capillary sampling with stylet slow-pull technique for intra-abdominal solid masses. J Clin Gastroenterol 2014; 49: 199-205

$35 \mathrm{Kim} \mathrm{GH}$, Cho YK, Kim EY et al. Comparison of 22-gauge aspiration needle with 22-gauge biopsy needle in endoscopic ultrasonography-guided subepithelial tumor sampling. Scandinavian J Gastro 2014; 49: $347-354$

36 Nagula S, Pourmand $K$, Aslanian $H$ et al. EUS-Fine Needle Aspiration (FNA) vs. EUS-Fine Needle Biopsy (FNB) for solid mass lesions: Interim analysis of a large multicenter, randomized clinical trial. DDW Orlando FL: 2013

37 Polkowski M, Larghi A, Weynand B et al. Learning, techniques, and complications of endoscopic ultrasound (EUS)-guided sampling in gastroenterology: European Society of Gastrointestinal Endoscopy (ESGE) Technical Guideline. Endoscopy 2012; 44: 190-206

38 Varadarajulu S, Hasan MK, Bang JY et al. Endoscopic ultrasound-guided tissue acquisition. Dig Endosc 2014; 26: (Suppl. 01) 62 -69

39 Eloubeidi MA, Varadarajulu S, Desai S et al. A prospective evaluation of an algorithm incorporating routine preoperative endoscopic ultrasound-guided fine needle aspiration in suspected pancreatic cancer. J Gastrointest Surg 2007; 11: 813-819 
40 Hewitt MJM, McPhail MJWM, Possamai LL et al. EUS-guided FNA for diagnosis of solid pancreatic neoplasms: a meta-analysis. Gastrointest Endosc 2012; 75: 319-3331

41 Chen G, Liu S, Zhao Y et al. Diagnostic accuracy of endoscopic ultrasound-guided fine-needle aspiration for pancreatic cancer: a meta-analysis. Pancreatology 2013; 13: 298-304

42 Puli SR, Bechtold ML, Buxbaum JL et al. How good is endoscopic ultrasound-guided fine-needle aspiration in diagnosing the correct etiology for a solid pancreatic mass? A meta-analysis and systematic review Pancreas 2013; 42: 20-26

43 Strand DS, Jeffus SK, Sauer BG et al. EUS-guided 22-gauge fine-needle aspiration versus core biopsy needle in the evaluation of solid pancreatic neoplasms. Diagn Cytopathol 2014; 42: 751 - 758

44 Vanbiervliet G, Napoléon B, Paul MCS et al. Core needle versus standard needle for endoscopic ultrasound-guided biopsy of solid pancreatic masses: a randomized crossover study. Endoscopy 2014; 46: 10631070
45 Lee YN, Moon JH, Kim HK et al. Core biopsy needle versus standard aspiration needle for endoscopic ultrasound-guided sampling of solid pancreatic masses: a randomized parallel-group study. Endoscopy 2014; 46: 1056-1062

46 Fujii LL, Levy MJ. Pitfalls in EUS FNA. Gastrointest Endosc Clin N Am 2014; $24: 125-142$

47 LeBlanc JK, Ciaccia D, Al-Assi MT et al. Optimal number of EUS-guided fine needle passes needed to obtain a correct diagnosis. Gastrointest Endosc 2004; 59: 475 - 481

48 Turner BG, Cizginer S, Agarwal D et al. Diagnosis of pancreatic neoplasia with EUS and FNA: a report of accuracy. Gastrointest Endosc 2010; 71: $91-98$

49 Wani S, Wallace MB, Cohen J et al. Quality indicators for EUS. Am J Gastroenterol 2015; 110: $102-113$

50 Affolter KE, Schmidt RL, Matynia AP et al. Needle size has only a limited effect on outcomes in EUS-guided fine needle aspiration: a systematic review and meta-analysis. Dig Dis Sci 2013; 58: 1026-1034 


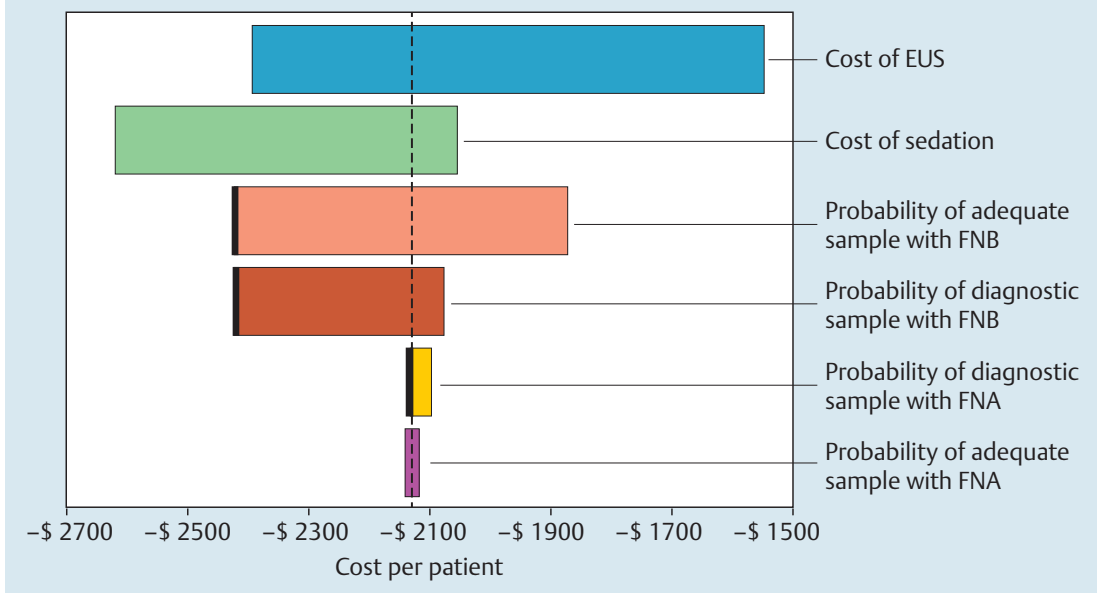

Supplementary Fig. 1 Tornado diagram examining the impact of important cost and outcome variables on the results of the decision analysis, with cost per patient along the $\mathrm{X}$ axis. In the tornado diagram, the uncertainty in the parameter associated with the largest bar, the one at the top of the chart has the maximum impact on the result, with each successive lower bar having a lesser impact. Also, thick vertical lines in the tornado diagram identify the threshold points where EUS-FNA becomes more economical (i. e. model conclusion is reversed). When the probability of adequate sampling by EUS-FNB falls below 0.38 , probability of diagnostic yield of EUS-FNB falls below 0.65 and the probability of diagnostic yield of EUS-FNA is higher than 0.87 . Similar results were noted for pancreatic and non-pancreatic masses.

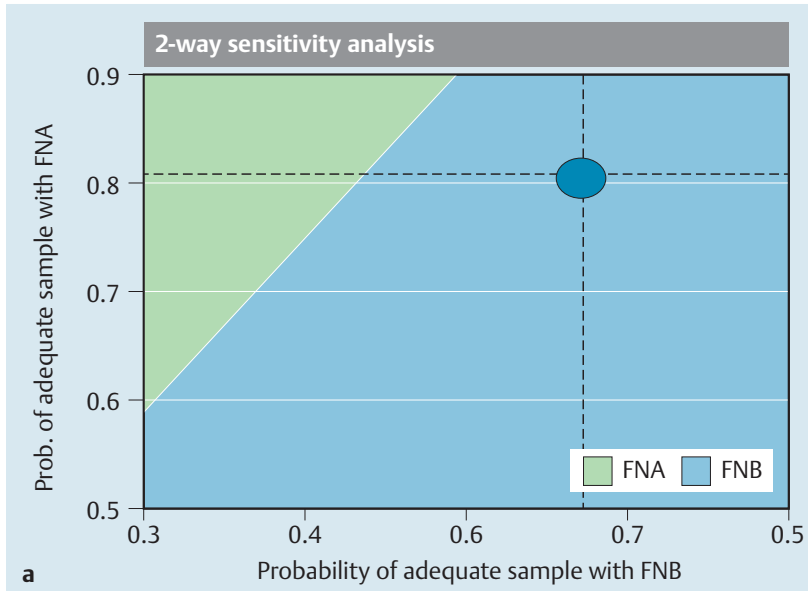

\begin{tabular}{|c|c|}
\hline Pancreatic lesions $(n=73)$ & $n(\%)$ \\
\hline Adenocarcinoma & $42(57.5)$ \\
\hline Pancreatic neuroendocrine tumor & $9(12.3)$ \\
\hline Metastatic adenocarcinoma & $9(12.3)$ \\
\hline Benign lymphoid cells (reactive LN) & $6(8.2)$ \\
\hline Abscess & $1(1.4)$ \\
\hline Chronic pancreatitis & $1(1.4)$ \\
\hline Leiomyoma & $1(1.4)$ \\
\hline Non-diagnostic & $4(5.5)$ \\
\hline Non-pancreatic lesions $(n=67)$ & $n(\%)$ \\
\hline Benign lymphoid cells (reactive LN) & $14(20.9)$ \\
\hline GIST & $12(17.9)$ \\
\hline B-cell Lymphoma & $6(9.0)$ \\
\hline \multicolumn{2}{|l|}{ Adenocarcinoma } \\
\hline Metastatic adenocarcinoma of unknown primary & $7(10.5)$ \\
\hline Metastatic pancreas adenocarcinoma & $4(6.0)$ \\
\hline Lung adenocarcinoma & $3(4.5)$ \\
\hline Gallbladder adenocarcinoma & $1(1.5)$ \\
\hline Metastatic colon adenocarcinoma & $1(1.5)$ \\
\hline Metastatic breast adenocarcinoma & $1(1.5)$ \\
\hline Leiomyoma/leiomyomasacroma & $3(4.5)$ \\
\hline Hepatocellular carcinoma & $2(3.0)$ \\
\hline Myxoid tumor & $1(1.5)$ \\
\hline Paraganglioma & $1(1.5)$ \\
\hline Abscess & $1(1.5)$ \\
\hline Pseudopapillary tumor & $1(1.5)$ \\
\hline Non-diagnostic & $9(13.4)$ \\
\hline
\end{tabular}

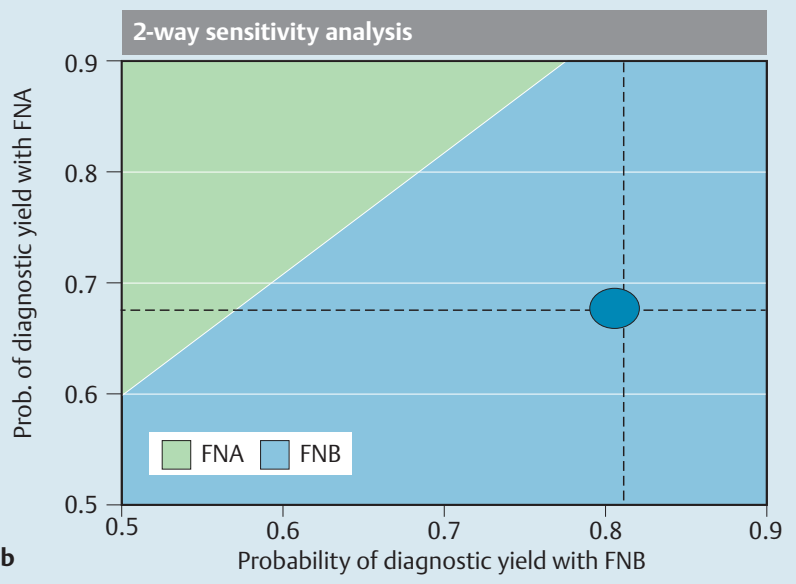

Supplementary Fig. $\mathbf{2} \mathbf{a}$ and $\mathbf{b}$ Results of a two-way sensitivity analysis with the $X$ axis showing probability of adequate sampling by EUS-FNB and the $Y$ axis showing probability of adequate sampling of EUS-FNA. When both these variables are simultaneously varied in the model, and the output of the model is plotted, any point in the blue shaded area favors EUSFNB-based strategy and any point in the green cross-hatched area favors EUS-FNA-based strategy. Similarly, Supplementary Fig. $\mathbf{2} \mathbf{b}$ shows the result of a two-way sensitivity analysis with the $X$ axis showing probability of diagnostic yield by EUS-FNB and the $Y$-axis showing probability of diagnostic yield of EUS-FNA. Blue circles in both figures represent when the data from the current RCT were plotted. It is evident that in a wide range of possibilities of these parameters around the point derived from this study, the EUS-FNB-based strategy is more economical. Similar results were noted for pancreatic and non-pancreatic masses. 